\title{
Financial Risk Information Spreading on Metapopulation Networks
}

\author{
Min Lin ${ }^{1}$ and Li Duan (iD) ${ }^{2}$ \\ ${ }^{1}$ School of Economics and Management, Sichuan Normal University, Chengdu 610101, China \\ ${ }^{2}$ Sichuan Academy of Social Sciences, Chengdu 610071, China \\ Correspondence should be addressed to Li Duan; dllr7673@sina.com
}

Received 15 December 2020; Revised 6 January 2021; Accepted 18 January 2021; Published 29 January 2021

Academic Editor: Wei Wang

Copyright (C) 2021 Min Lin and Li Duan. This is an open access article distributed under the Creative Commons Attribution License, which permits unrestricted use, distribution, and reproduction in any medium, provided the original work is properly cited.

\begin{abstract}
The financial risk information diffuses through various kinds of social networks, such as Twitter and Facebook. Individuals transmit the financial risk information which can migrate among different platforms or forums. In this paper, we propose a financial risk information spreading model on metapopulation networks. The subpopulation represents a platform or forum, and individuals migrate among them to transmit the information. We use a discrete-time Markov chain approach to describe the spreading dynamics' evolution and deduce the outbreak threshold point. We perform numerical simulation on artificial networks and discover that the financial risk information can be promoted once increasing the information transmission probability and active subpopulation fraction. The weight variance and migration probability cannot significantly affect the financial risk spreading size. The discrete-time Markov chain approach can reasonably predict the above phenomena.
\end{abstract}

\section{Introduction}

Many real-world systems in society, economy, and biological systems can be described as complex networks [1-3]. The nodes represent the element, and edges stand for the relationships among nodes. For instance, in financial network, the nodes stand for the financial institution, e.g., banks, and edges means the loan relationships among those financial institutions [4-6]. With such description framework, the dynamics of financial behavior, risk spreading can be mapped into studying the dynamics on financial networks [7-13]. Gai et al. [7] studied the risk contagion on directed financial networks and assumed that a bank's failure could trigger its lenders and further induce the cascading failures. Garas et al. [14] used a susceptible-infected-recovered (SIR) model to describe the economic crisis on the financial network and revealed that Belgium could initiate a global crisis.

In reality, the information about the financial risk is always accompanied by the financial risk [15]. That information widely spreads on various kinds of social platforms and forums, such as Twitter and Facebook. To describe the information spreading dynamics, some successful models have been proposed, such as the epidemic susceptible-infected-susceptible [16, 17], susceptible-infected-recovered [18, 19], Watts threshold model [20], and independent cascading [21]. Researchers have demonstrated that the network topologies markedly affect the spreading dynamics [22-28]. While spreading dynamics on networks with heterogeneous degree distribution, the strong heavy-tail may induce the outbreak threshold to disappear. When the information spreads on different platforms and forums, scholars are modeled as multiplex networks [29-33] or metapopulation networks [34-36]. Compared with spreading dynamics on single networks, multiplex networks can promote or suppress the spreading, which depends on the evolutionary mechanisms [37-39]. Recently, Gomez et al. [40] studied the phase diagram of the information spreading on metapopulation networks and revealed that mobility of individuals in large-size 
subpopulations towards smaller ones suppress the spreading.

Real-data analysis has revealed that different platforms and forums usually have a different attitude on the same piece of information. Some exhibit enthusiasm in the information, while others demonstrate depression. To include this factor, Wang et al. [41] proposed an informationspreading model on heterogeneous multiplex networks and discussed the system's spreading size and critical points. There is still a mathematical model to describe the financial risk information-spreading dynamics on heterogeneous metapopulation networks to our vast knowledge. We, therefore, propose a model in Section 2. Then, we develop a discrete-time Markov chain approach to study the evolution of financial risk information spreading on metapopulation in Section 3. The numerical simulations of the spreading dynamics are performed in Section 4. Finally, we make conclusions in Section 5.

\section{Model Descriptions}

In this section, we propose information spreading model on metapopulation, which includes $N$ subpopulation. In each subpopulation $i$, there are $n_{i}$ aboriginal (nodes). An edge between two subpopulations represents a physical or cybernetic connection that exists. The edge's wight stands for the strength between the two subpopulations, which can be used to describe the interaction strength between different platforms or forums. In this paper, we build the interconnections among subpopulation according to a given degree distribution:

$$
P(k)=\frac{\langle k\rangle^{k}}{k !} \exp (-\langle k\rangle),
$$

where $\langle k\rangle$ is the average neighbor subpopulation. Assume the edge weight distribution

$$
G(w)=\frac{1}{\sqrt{2 \pi \sigma}} \exp \left(-\frac{(w-\langle w\rangle)^{2}}{2 \sigma^{2}}\right)
$$

where $\langle w\rangle$ is the average weight and $\sigma$ is the standard variance. An illustration of the metapopulation is shown in Figure 1. Mathematically, the metapopulation's network topology can be described by using the weighted adjacency matrix $W$, in which $W_{i j}=1$ means that subpopulation $i$ and $j$ are connected by an edge with strength $W_{i j}$. In reality, each subpopulation has its characters in spreading dynamics. To include this character, we classify the subpopulation into two types: active subpopulation (AS) and

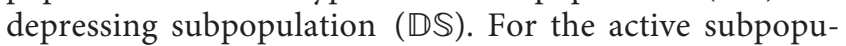
lation, the individuals actively participate and transmit the information. On the contrary, individuals in depressing subpopulation are less active in transmitting the information. To obtain a mathematical result, we assume a fraction $\alpha$ of (AS) and $1-\alpha$ fraction of (DSS). At every time step, each individual will prefer to move to a neighboring subpopulation with probability $p_{d}$. The preference probability of an individual in subpopulation $i$ moving to subpopulation $j$ is as follows:

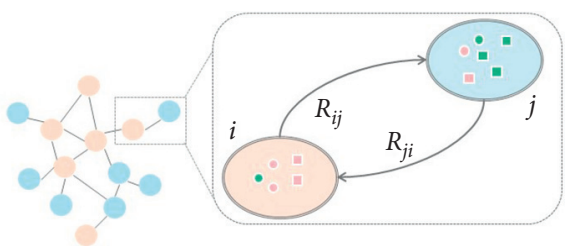

FIgURE 1: Illustration of financial risk information spreading on metapopulation network. Orange and blue circles represent the depressing subpopulation $(\mathbb{D S})$ and active subpopulation $(\mathbb{A} \mathbb{S}$ ), respectively. Green and blue circles stand for the unaware and aware individuals. The directed edges $R_{i j}$ means the preference travelling probability from subpopulation $i$ to $j$.

$$
R_{i j}=\frac{W_{i j}}{\sum_{l=1}^{N} W_{i l}} .
$$

At the end of each time step, each travelling individual returns to his/her place of residence.

To describe the financial risk information spreading dynamics, we adopt an unaware-aware-unaware (UAU) model $[42,43]$. The unaware individual means that an individual does not know the financial risk information and maybe obtain the financial risk information in the following steps. An aware individual who stands for himself/herself has obtained the information and is willing to share it with others in the same subpopulation. The financial risk information spreading dynamics evolves as follows. Initially, we randomly select $\varrho n_{i}$ individuals as the seeds of the spreading, i.e., set those $\varrho n_{i}$ individuals in the aware state. At every time step, we perform the following two processes sequentially. (i) Each individual in each subpopulation moves with probability $p_{d}$, and his/her determination selection probability is determined according to equation (3). This migration can be regarded as an individual move from one forum (or communication group) to another. Individuals in the same subpopulation are sharing financial risk information. In (ASS), e.g., subpopulation $i$, each active individual will randomly contact $\widetilde{k}$ individuals and try to transmit the financial risk information to each unaware individuals of the $\widetilde{k}$ individuals with probability $\lambda$. For the unaware individual, he/she becomes aware if he/she receives at least one piece of financial risk information from neighbors successfully. The contagion process in the depressing subpopulation (DS) is similar to that in the AS. The only difference is that an unaware individual becoming aware should receive at least $\theta$ pieces of information from aware neighbors successfully. (ii) The travelling individuals return to his/her residence, and each aware individual becomes unaware with probability $\mu$. The spreading dynamics evolve until the system reaches a dynamic steady state.

\section{Theoretical Analysis}

Inspired by [40, 44, 45], we develop a discrete-time Markov chain approach to study the evolution of financial risk information spreading on metapopulation. In theory, we assume that the infection probability of unaware node is independent, that is to say, there are no dynamical 
correlations. Denote $\rho_{i}(t)(i=1, \ldots, N)$ as the fraction of active individuals in subpopulation $i$ at time $t$. The evolution of $\rho_{i}(t)$ can be expressed as

$$
\rho_{i}(t+1)=(1-\mu) \rho_{i}(t)+\left(1-\rho_{i}(t)\right) \Theta_{i}(t) .
$$

On the right-hand side of equation (4), the first expression represents the fraction of active individuals who do not recover. The second expression stands for the probability that the unaware individuals become aware. The expression $\Theta_{i}(t)$ means an unaware individual in subpopulation $i$ is infected by active individuals.

Once the expression of $\Theta_{i}(t)$ is obtained, we know the evolution of $\rho_{i}(t)$. In the following, we solve the expression of $\Theta_{i}(t)$. An unaware individual $b_{i}$ in subpopulation $i$ becoming aware has two situations. (i) If individual $b_{i}$ does not travel to neighboring subpopulation, he/she will be infected at time $t$ in his own aboriginal subpopulation $i$ with probability:

$$
P_{i}(t)=\alpha P_{i}^{1}(t)+(1-\alpha) P_{i}^{2}(t) .
$$

The first expression on the right hand of equation (5) stands for the contagion probability when subpopulation $i$ is AS. The second expression represents the contagion probability when the subpopulation $i$ is $(\mathbb{D S})$. When the

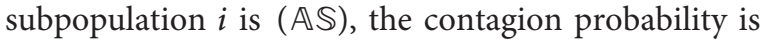

$$
P_{i}^{1}(t)=1-(1-\lambda)^{x_{i}(t) \tilde{k}}
$$

where $x_{i}(t)$ stands for the fraction of active individuals in subpopulation $i$ after the migration, and the expression of $x_{i}(t)$ is

$$
\begin{aligned}
x_{i}(t) & =\frac{\sum_{j=1}^{N} n_{j \longrightarrow i} \rho_{j}(t)}{\sum_{j=1}^{N} n_{j \longrightarrow i}} \\
& =\frac{\sum_{j=1}^{N} n_{j \longrightarrow i} \rho_{j}(t)}{n_{i}^{\text {eff }}},
\end{aligned}
$$

where $n_{i}^{\text {eff }}=\sum_{j} n_{j \longrightarrow i}$ is the number of effective individuals in subpopulation $i$ after migration. The term $n_{j \longrightarrow i}$ represents the number of individuals travelling from subpopulation $j$ to subpopulation $i$ and can be expressed as

$$
n_{j \longrightarrow i}=\delta_{i j}\left(1-p_{d}\right) n_{i}+p_{d} \frac{W_{j i}}{\sum_{l=1}^{N} W_{j l}} n_{j}
$$

where $\delta_{i j}=1$ when $i=j$; otherwise, $\delta_{i j}=0$. In equation (8), the first second term stands for the number of individuals that do not travel to neighboring subpopulation, and the second term represents the number of individuals in which individuals in neighbors' subpopulation travelling to subpopulation $i$. (ii) If individual $b_{i}$ travels to neighboring subpopulation and is infected at his/her determination, the contagion probability is $p_{d} \sum_{j=1}^{N} h_{i \rightarrow j} P_{j}(t)$. Combining the above stated two situations, we obtain the expression of $\Theta_{i}(t)$ as

$$
\Theta_{i}(t)=\left(1-p_{d}\right) P_{i}(t)+p_{d} \sum_{j=1}^{N} \frac{W_{i j}}{\sum_{l=1}^{N} W_{i l}} P_{j}(t) .
$$

The above equations describe the financial risk information spreading dynamics on metapopulation. Iterating equation (4) from an initial value of $\rho_{i}(t)$, we obtain the dynamical steady state of $\rho_{i}(t \longrightarrow \infty)$. When the number of subpopulation is large, numerically solving the equations is hard, and we may use the dimensionality reduction approach. For the sake of simplicity, we denote $\rho_{i}(t \longrightarrow \infty)$ as $\rho_{i}^{*}$. The average fraction of individuals in the active state in the steady state is as

$$
\langle\rho\rangle=\frac{1}{N} \sum_{i=1}^{N} \rho_{i}^{*}
$$

The values of $\langle\rho\rangle$ can be regarded as the order parameter of the system in statistical physics. That is to say, the global financial risk information outbreaks, i.e., $\langle\rho\rangle>0$, when the information transmission probability $\lambda$ is above a critical value $\lambda_{c}$. Otherwise, only a vanishingly small fraction of individuals is in the active state, i.e., $\langle\rho\rangle=0$. In the following, we solve the expression of $\lambda_{c}$. In the steady state, we know $\rho_{i}(t+1)=\rho_{i}(t)=\rho_{i}^{*}$; therefore, equation (4) can be rewritten as

$$
\mu \rho_{i}^{*}=\left(1-\rho_{i}^{*}\right)\left[\left(1-p_{d}\right) P_{i}+p_{d} \sum_{j=1}^{N} \frac{W_{i j}}{\sum_{l=1}^{N} W_{i l}} P_{j}\right],
$$

where

$$
\begin{aligned}
P_{i}= & \alpha P_{i}^{1}+(1-\alpha) P_{i}^{2} \\
= & \alpha\left(1-(1-\lambda)^{x_{i} \tilde{k}}\right)+(1-\alpha) \\
& \cdot\left[1-\left(\sum_{m=0}^{\theta-1}(1-\lambda)^{x_{i} \tilde{k}-m} \lambda^{m}\left(\begin{array}{c}
x_{i} \tilde{k} \\
m
\end{array}\right)\right)\right] .
\end{aligned}
$$

Near the critical value $\lambda_{c}$, the information spreading size is very small, i.e., $\rho_{i}^{*} \ll 1$; thus, we neglect the high orders of $\rho_{i}^{*}$. In addition, we assume that $\lambda$ is small enough and obtain $(1-\lambda)^{n} \simeq 1-n \lambda$. Linearizing equation (12), we have $P_{i} \simeq \alpha x_{i} k \lambda$. Using this term, we further obtain

$$
\Theta_{i} \simeq\left(1-p_{d}\right) \alpha x_{i} \tilde{k} \lambda+p_{d} \sum_{j=1}^{N} R_{i j} \alpha x_{j} \tilde{k} \lambda .
$$

Inserting equations (7) and (8) into the above equation, we have

$$
\begin{aligned}
\Theta_{i} \simeq\left(1-p_{d}\right) \alpha k \lambda \frac{\left(1-p_{d}\right) n_{i} \varepsilon_{i}^{*}+p_{d} \sum_{j=1}^{N} R_{j i} n_{j} \varepsilon_{j}^{*}}{\left(1-p_{d}\right) n_{i}+p_{d} \sum_{j=1}^{N} R_{j i} n_{j}} \\
\quad+p_{d} \alpha k \lambda \sum_{j=1}^{N} R_{i j} \frac{\left(1-p_{d}\right) n_{j} \varepsilon_{j}^{*}+p_{d} \sum_{l=1}^{N} R_{l j} n_{l} \varepsilon_{l}^{*}}{\left(1-p_{d}\right) n_{j}+p_{d} \sum_{l=1}^{N} R_{l j} n_{l}} .
\end{aligned}
$$

During the computing process, we used the relation $\sum_{j=1}^{N} \delta_{i j} \varepsilon_{j}^{*}=\varepsilon_{i}^{*}$. We further obtain 


$$
\begin{aligned}
& \Theta \simeq\left(1-p_{d}\right) \alpha k \lambda\left(\mathbb{M}^{1}+\mathbb{M}^{2} R^{T} \mathbb{Z}\right) \varepsilon^{*}+p_{d} \alpha k \lambda R\left(\mathbb{M}^{1}+\mathbb{M}^{2} R^{T} \mathbb{U}\right) \varepsilon^{*} \\
& =\alpha k \lambda\left(\mathbb{M}^{1}+\mathbb{M}^{2} R^{T} \mathbb{U}\right) \varepsilon^{*} .
\end{aligned}
$$

An element of matrix $\mathbb{M}^{1}$ is

$$
\mathbb{M}_{i j}^{1}= \begin{cases}0, & i \neq j, \\ \frac{\left(1-p_{d}\right) n_{i}}{\left(1-p_{d}\right) n_{i}+p_{d} \sum_{l=1}^{N} R_{l i} n_{l}}, & i=j .\end{cases}
$$

The element of matrix $\mathbb{M}^{2}$ is

$$
\mathbb{M}_{i j}^{2}= \begin{cases}0, & i \neq j, \\ \frac{p_{d} n_{i}}{\left(1-p_{d}\right) n_{i}+p_{d} \sum_{l=1}^{N} h_{l \longrightarrow i} n_{l}}, & i=j .\end{cases}
$$

The element of matrix $\mathbb{H}$ is

$$
\mathbb{H}_{i j}= \begin{cases}0, & i \neq j, \\ n_{i}, & i=j .\end{cases}
$$

Denoting $\mathbb{M}=\alpha k\left(\mathbb{M}^{1}+\mathbb{M}^{2} R^{T} \mathfrak{H}\right)$, we have

$$
\frac{\mu}{\lambda} \varepsilon^{*}=\mathbb{M} \varepsilon^{*}
$$
is

The global financial risk information outbreak condition

$$
\lambda_{c}=\frac{\mu}{\Lambda_{\max }(\mathbb{M})}
$$

where $\Lambda_{\max }(\mathbb{M})$ is the largest eigenvalue of matrix $\mathbb{M}$. Numerically, solving equation (20), we obtain the value of $\lambda_{c}$.

\section{Numerical Results' Analysis}

To systemically investigate the financial risk information spreading dynamics on metapopulation networks, we perform extensive numerical simulations. We generate $N=50$ subpopulation. For each subpopulation $i$, we set $n_{i}=100$ number of residents. We randomly connect the 50 subpopulations with a given probability of $u=0.3$. As a result, the average connection of a subpopulation is $\langle k\rangle=15$. The edge weight distribution is $G(w)$ with average weight $\langle w\rangle=10$. For the $\mathbb{D} S$, we set the $\theta=3$. That is to say, an unaware individual in a $\mathbb{D S}$ becoming aware must receive three pieces of information successfully at a given time step. Through extensive numerical simulations, we found that other values of $\theta$ do not affect the results qualitatively. We perform numerical simulations at least 200 times when the system reaches a steady state for a given parameter set. We found that the spreading dynamics reached a steady state after 500 time steps through performing extensive numerical simulations. Mathematically, we verify that the system reaches a steady state when

$$
\delta \rho=|\widehat{\rho}(t+1)-\widehat{\rho}(t)|
$$

is small than $\varepsilon=10^{-5}$, where $\hat{\rho}(t)=\sum_{t^{\prime}=t-100}^{t} \rho(t \prime)$.

In Figure 2, we investigate the effects of four key parameters on the financial risk information spreading on metapopulation networks. We first discuss the impact of information transmission probability. As shown in Figures 2(a) and 2(b), we present the average fraction $\langle\rho\rangle$ of individuals in the aware state as a function of information transmission probability $\lambda$. We reveal that increasing the values of $\lambda$ is beneficial for information spreading, i.e., $\langle\rho\rangle$ with $\lambda$. When $\lambda \leq \lambda_{c}$, which can be obtained by numerically solving equation (20), there are no individuals who received the financial risk information, that is, $\langle\rho\rangle=0$. When $\lambda>\lambda_{c}$, we find that $\langle\rho\rangle$ is a finite value, which indicates the global financial risk information outbreak. This phenomenon demonstrates that we can contain the financial risk information spreading by reducing the information transmission probability.

We now discuss the effects of the second key parameter, fraction of $\mathbb{A D}$, on the spreading dynamics. As shown in Figure 2(a), we find that increasing the fraction of $\mathbb{A D}$ promotes the information spreading. That is to say, $\langle\rho\rangle$ increases with $\alpha$. For large values of $\alpha$, there are more $\mathbb{A} \mathbb{D}$. Therefore, more unaware individuals becoming aware only need to receive one piece of information from others. As a result, the global financial risk information spreading dynamics is promoted.

The third key parameter is the variance of the weight distribution. In Figure 1, we set $\sigma^{2}=0$, 4, and 9 for different values of $\alpha$ and $p_{d}$. We reveal that the variance does not markedly affect the financial risk information spreading size and outbreak threshold. This phenomenon indicates that the varying connection strength among subpopulations cannot significantly affect the spreading of financial risk. Lastly, we revealed that the travelling probability does not significantly affect the spreading dynamics. Our proposed discrete-time Markov chain approach can predict the above phenomena.

Finally, we study the financial risk information spreading size as a function of travelling probability $p_{d}$ and information transmission probability $\lambda$ in detail in Figure 3. For small values of $\alpha$, i.e., a few subpopulations are $\mathbb{A} S$, the financial risk information cannot globally outbreak for any values of $p_{d}$ and $\lambda$, as shown in Figures 3(a) and 3(b). With 


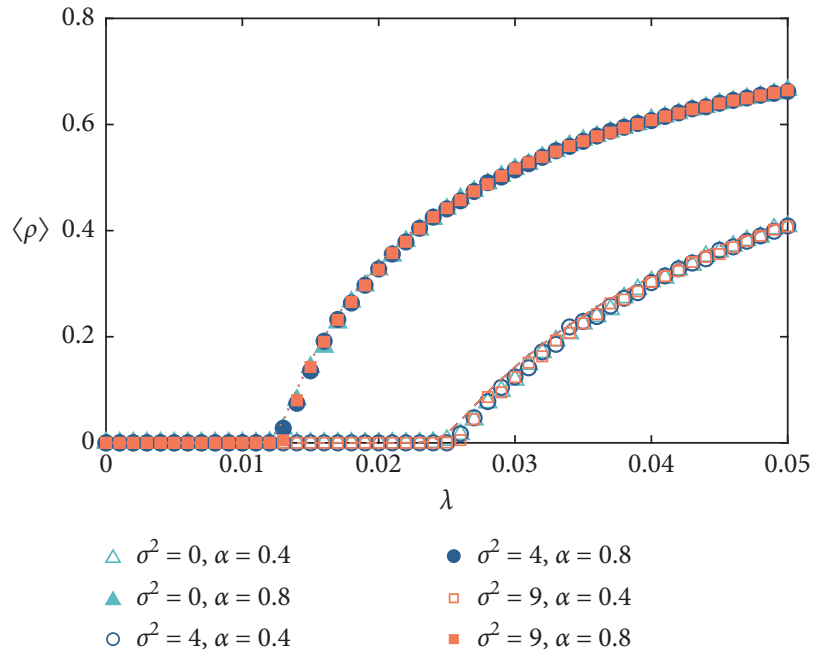

(a)

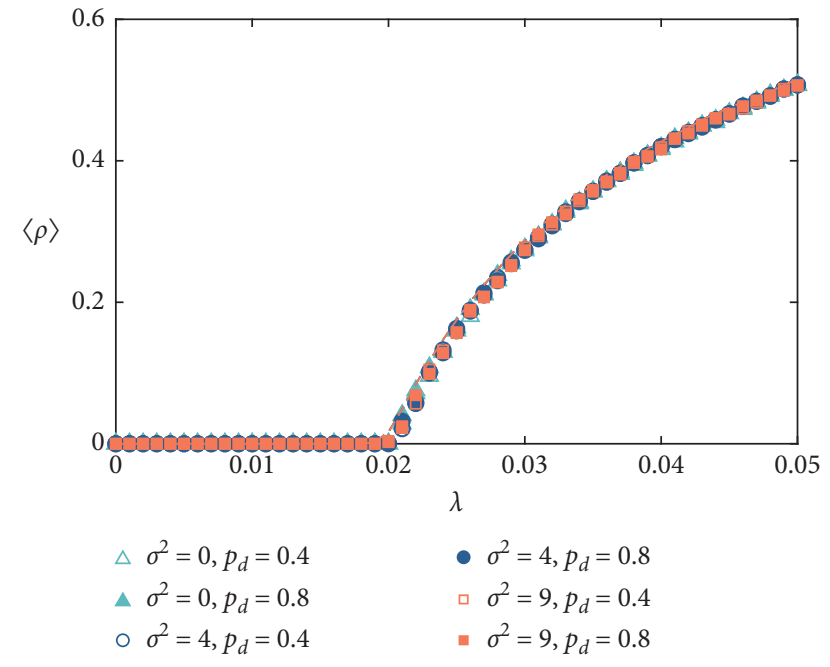

(b)

FIGURE 2: Financial risk information spreading on metapopulation networks. (a) The fraction of individuals in the active state $\langle\rho\rangle$ versus information transmission probability $\lambda$ with travelling probability $p_{d}=0.5$. (b) $\langle\rho\rangle$ versus $\lambda$ with $\alpha=0.5$. The average weight is set to be $\langle w\rangle=10$ and recovery probability $\mu=0.2$. The lines stand for the theoretical analysis and symbols represent the numerical simulation results.

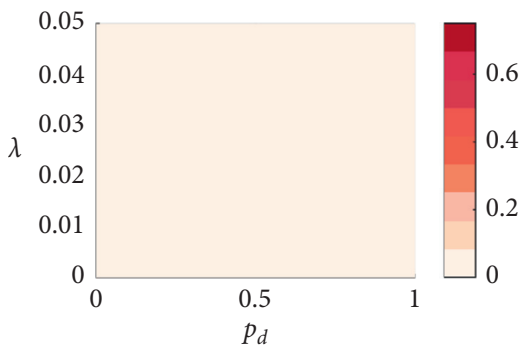

(a)

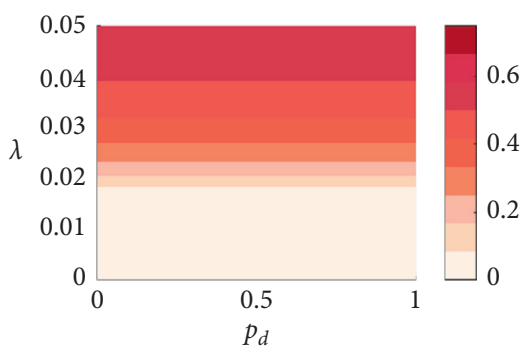

(d)

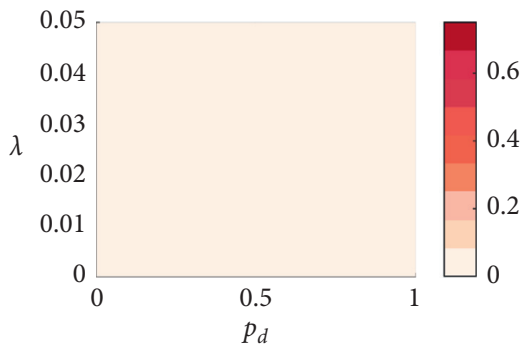

(b)

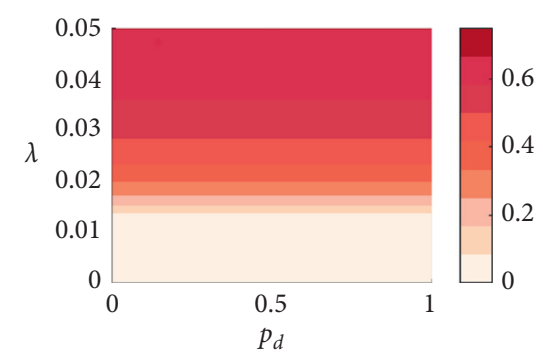

(e)

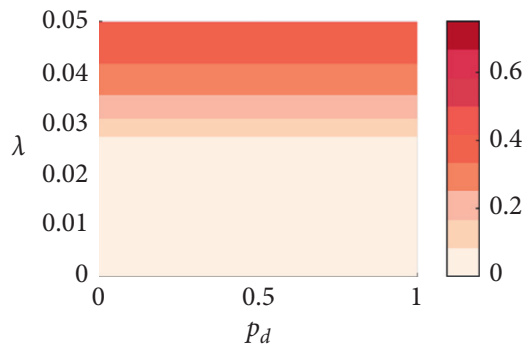

(c)

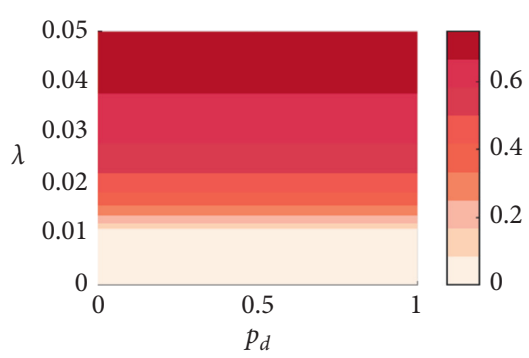

(f)

FIGURE 3: The financial risk information spreading on metapopulation on $\left(p_{d}, \lambda\right)$ plane. The financial risk information spreading size $\langle\rho\rangle$ as a function of travelling probability $p_{d}$ and information transmission probability $\lambda$ with $\alpha=0$ (a), $\alpha=0.2$ (b), $\alpha=0.4$ (c), $\alpha=0.6$ (d), $\alpha=0.8$ (e), and $\alpha=1$ (f). Different colors represent the values of $\langle\rho\rangle$.

the increase of $\alpha$, the global financial risk information outbreak becomes possible when $\lambda>\lambda_{c}$. In Figures 3(c)-3(f), we find that $\langle\rho\rangle$ does not vary with $p_{d}$.

\section{Conclusions}

In this paper, we investigated the financial risk information spreading on social networks. To describe the different social platforms and forums in the spreading dynamics, we adopt the metapopulation network, in which a forum is a subpopulation and mixed randomly. At every time step, each individual may travel from one subpopulation to another. We used a discrete-time Markov chain approach to describe the dynamical process and critical point. Our theory can reasonably predict numerical simulations. We noted that the financial risk information spreading size depends on the 
information transmission probability, a fraction of active subpopulation, and independent on the variance of the weight distribution and travelling probability. Our results may shed some light on studying the financial risk information spreading dynamics and contain the financial risk. On the one hand, our model and theoretical approach maybe used to study other dynamics on metapopulation networks. On the other hand, to contain the financial risk information spreading, we can reduce the information transmission probability. For more realistical situations, the fraction of DS is not simply following a binomial distribution. Therefore, other forms of distribution need further studies.

\section{Data Availability}

The data that support the findings of this study are available from the corresponding author upon request.

\section{Conflicts of Interest}

The authors declare that they have no conflicts of interest.

\section{Acknowledgments}

This work was partially supported by Sichuan Science and Technology Program (no. 2020JDR0200) and National Social Science Foundation of China Western Project (no. 18XJY001).

\section{References}

[1] R. Albert and A.-L. Barabási, "Statistical mechanics of complex networks," Reviews of Modern Physics, vol. 74, no. 1, p. 47, 2002.

[2] M. Newman, Networks, Oxford University Press, Oxford, UK, 2018.

[3] S. N. Dorogovtsev and J. F. F. Mendes, "Evolution of networks," Advances in Physics, vol. 51, no. 4, p. 1079, 2002.

[4] A. G. Haldane and R. M. May, "Systemic risk in banking ecosystems," Nature, vol. 469, no. 7330, p. 351, 2011.

[5] A. G. Haldane, Fragile Stabilität-Stabile Fragilität, pp. 243-278, Springer, Berlin, Germany, 2013.

[6] P. Barucca, M. Bardoscia, F. Caccioli et al., "Network valuation in financial systems," Mathematical Finance, vol. 30, no. 4, pp. 1181-1204, 2020.

[7] P. Gai and S. Kapadia, "Contagion in financial networks," Proceedings of the Royal Society A: Mathematical, Physical and Engineering Sciences, vol. 466, no. 2120, p. 2401, 2010.

[8] Z. Wang, X. Gao, H. An, R. Tang, and Q. Sun, "Identifying influential energy stocks based on spillover network," International Review of Financial Analysis, vol. 68, pp. 101-277, 2020.

[9] F. Linardi, C. Diks, M. Van Der Leij, and I. Lazier, "Dynamic interbank network analysis using latent space models," Journal of Economic Dynamics and Control, vol. 112, Article ID 103792, 2020.

[10] M. Elliott, B. Golub, and M. O. Jackson, "Financial networks and contagion," American Economic Review, vol. 104, no. 10, p. $3115,2014$.

[11] R. Cont, A. Moussa, and E. B. e Santos, "Network structure and systemic risk in banking systems," SSRN Electronic Journal, 2010.
[12] S. Khanna and B. Hemenway Falk, Jacobs Levy Equity Management Center for Quantitative Financial Research Paper, University Of Pennsylvania, Philadelphia, PA 19104, USA, 2020.

[13] R. Doyle, "Transparency in language generation: levels of automation," 2020, https://arxiv.org/abs/2006.06295.

[14] A. Garas, P. Argyrakis, C. Rozenblat, M. Tomassini, and S. Havlin, "Worldwide spreading of economic crisis," New Journal of Physics, vol. 12, no. 11, p. 113043, 2010.

[15] Z.-K. Zhang, C. Liu, X.-X. Zhan, X. Lu, C.-X. Zhang, and Y.-C. Zhang, "Dynamics of information diffusion and its applications on complex networks," Physics Reports, vol. 651, p. 1, 2016.

[16] R. Pastor-Satorras and A. Vespignani, "Epidemic dynamics and endemic states in complex networks," Physical Review E, vol. 63, Article ID 066117, 2001.

[17] R. Pastor-Satorras and A. Vespignani, "Immunization of complex networks," Physical Review E, vol. 65, Article ID 035108, 2002.

[18] Y. Moreno, R. Pastor-Satorras, and A. Vespignani, "When individual behaviour matters: homogeneous and network models in epidemiology," The European Physical Journal B, vol. 26, no. 4, p. 521, 2002.

[19] W. Wang, M. Tang, H.-F. Zhang, H. Gao, Y. Do, and Z.-H. Liu, "Epidemic spreading on complex networks with general degree and weight distributions," Physical Review E, vol. 90, Article ID 042803, 2014.

[20] D. J. Watts, "A simple model of global cascades on random networks," Proceedings of the National Academy of Sciences, vol. 99, no. 9, pp. 57-66, 2002.

[21] W. Chen, Y. Wang, and S. Yang in Proceedings of the 15th ACM SIGKDD International Conference on Knowledge Discovery and Data Mining, pp. 199-208, June 2009.

[22] C. Castellano, S. Fortunato, and V. Loreto, "Statistical physics of social dynamics," Reviews of Modern Physics, vol. 81, no. 2, p. 591, 2009.

[23] C. Castellano and R. Pastor-Satorras, "Thresholds for epidemic spreading in networks," Physical Review Letters, vol. 105, Article ID 218701, 2010.

[24] W. Wang, M. Tang, H. E. Stanley, and L. A. Braunstein, "Unification of theoretical approaches for epidemic spreading on complex networks," Reports on Progress in Physics, vol. 80, no. 80, p. 36603, 2017.

[25] M. Boguná, R. Pastor-Satorras, and A. Vespignani, “Absence of epidemic threshold in scale-free networks with degree correlations," Physical Review Letters, vol. 90, Article ID 028701, 2003.

[26] W. Wang, Q.-H. Liu, J. Liang, Y. Hu, and T. Zhou, "Coevolution spreading in complex networks," Physics Reports, vol. 820, p. 1, 2019.

[27] M. Starnini, J. P. Gleeson, and M. Boguñá, "Equivalence between non-markovian and markovian dynamics in epidemic spreading processes," Physical Review Letters, vol. 118, Article ID 128301, 2017.

[28] D.-W. Huang, L.-X. Yang, X. Yang, Y. Y. Tang, and J. Bi, "Discrete dynamics in nature and society 2020," Discrete Dynamics in Nature and Society (DDNS), 2020.

[29] M. Kivelä, A. Arenas, M. Barthelemy, J. P. Gleeson, Y. Moreno, and M. A. Porter, "Multilayer networks," Journal of Complex Networks, vol. 2, no. 3, p. 203, 2014.

[30] M. De Domenico, V. Nicosia, A. Arenas, and V. Latora, "Structural reducibility of multilayer networks," Nature Communications, vol. 6, p. 1, 2015. 
[31] S. Boccaletti, G. Bianconi, R. Criado et al., "The structure and dynamics of multilayer networks," Physics Reports, vol. 544, no. 1, p. 1, 2014.

[32] M. De Domenico, A. Solé-Ribalta, E. Cozzo et al., "Mathematical formulation of multilayer networks," Physical Review X, vol. 3, Article ID 041022, 2013.

[33] K.-M. Lee, B. Min, and K.-I. Goh, "Towards real-world complexity: an introduction to multiplex networks," The European Physical Journal B, vol. 88, p. 48, 2015.

[34] V. Colizza, R. Pastor-Satorras, and A. Vespignani, "Reactiondiffusion processes and metapopulation models in heterogeneous networks," Nature Physics, vol. 3, no. 4, p. 276, 2007.

[35] L. Wang and X. Li, "Spatial epidemiology of networked metapopulation: an overview," Chinese Science Bulletin, vol. 59, no. 28, p. 3511, 2014.

[36] S.-Y. Liu, A. Baronchelli, and N. Perra, "Contagion dynamics in time-varying metapopulation networks," Physical Review E, vol. 87, Article ID 032805, 2013.

[37] B. Min, S.-H. Gwak, N. Lee, and K.-I. Goh, "Layerswitching cost and optimality in information spreading on multiplex networks," Scientific Reports, vol. 6, Article ID 21392, 2016.

[38] Z. Wang, C. Xia, Z. Chen, and G. Chen, Institute of Electrical and Electronics Engineers Transactions on Cybernetics, 2020.

[39] H. Liang'an, H. Guo, Y. Cheng, and X. Xie, "A new model for supply chain risk propagation considering herd mentality and risk preference under warning information on multiplex networks," Physica A: Statistical Mechanics and Its Applications, vol. 545, Article ID 123506, 2020.

[40] J. Gómez-Gardeñes, D. Soriano-Paños, and A. Arenas, "Critical regimes driven by recurrent mobility patterns of reaction-diffusion processes in networks," Nature Physics, vol. 14, no. 4, p. 391, 2018.

[41] W. Wang, X.-L. Chen, and L.-F. Zhong, "Social contagions with heterogeneous credibility," Physica A: Statistical Mechanics and Its Applications, vol. 503, p. 604, 2018.

[42] C. Granell, S. Gómez, and A. Arenas, "Dynamical interplay between awareness and epidemic spreading in multiplex networks," Physical Review Letters, vol. 111, Article ID 128701, 2013.

[43] E. H. Xu, W. Wang, C. Xu, M. Tang, Y. Do, and P. Hui, "Suppressed epidemics in multirelational networks," Physical Review E, vol. 92, Article ID 022812, 2015.

[44] S. Gómez, A. Arenas, J. Borge-Holthoefer, S. Meloni, and Y. Moreno, "Discrete-time Markov chain approach to contact-based disease spreading in complex networks," EPL (Europhysics Letters), vol. 89, no. 3, p. 38009, 2010.

[45] W. Wang, Y. Ma, T. Wu, Y. Dai, X. Chen, and L. A. Braunstein, "Containing misinformation spreading in temporal social networks," Chaos: An Interdisciplinary Journal of Nonlinear Science, vol. 29, no. 12, pp. 123-131, 2019. 